\title{
Distortion Invariant Object Recognition Based on Mach- Zehnder Joint Transform Correlation in YIQ Colour Space
}

\author{
Chulung Chen, Kaining Gu, Jianshuen Fang, Chungcheng Lee \\ Department of Electrical Engineering, Yuan Ze University, Taiwan; \\ chulung@saturn.yzu.edu.tw
}

\begin{abstract}
We investigate the YIQ colour space in conjunction with average cross correlation optimization algorithm to design the reference function for pattern recognition on various views of the interested colour object. Joint transform correlation is devoted for recognition of colour targets. The reference function for each color channel is trained with true class images rotated in-plane at 2 degrees intervals between -14 degrees and 14 degrees. Results indicates the feasibility of our proposed method.
\end{abstract}

Keywords: Joint transform correlation; Colour pattern recognition; YIQ colour space.

\section{Introduction}

VanderLugt correlator (VLC) [1] and the joint transform correlator (JTC) [2] have already been two well known optical correlators. VLC was proposed for comparing two signals by utilising the Fourier transforming properties of a lens. This is the most commonly used type of correlator, but perhaps the most complex and sensitive to build, due to its strict alignment criteria. In 1966, Weaver and Goodman introduced the JTC for pattern recognition application. A few years later, LCD based JTC [3] proposed by $\mathrm{Yu}$ and Lu became an attractive tool for pattern recognition. Since then, the JTC configuration has received increased attention because the JTC does not require a complex filter in the Fourier plane like the VLC. However, the classical JTC yields poor correlation output where a large zero-order term (also called DC term) dominates the correlation outputs. The DC term is the sum of each autocorrelation of the reference image and the target image at the output of correlation plane. The existence of the DC term will influence the performance, therefore the removal of the nonzero-order term is of great importance.

To deal with the DC term, Lu et al. [4] adoped phase-shifting technique to design a nonzero- order JTC (NOJTC) and Li et al. [6] used the joint transform power spectrum (JTPS) subtraction strategy to realize the NOJTC. The Mach-Zehnder JTC (MZJTC) [6-8] can remove the zero-order term in only one step directly without storing the Fourier spectra of both the reference and target images beforehand. Later, Chen et al. $[9,10]$ adopted constraint optimization based on Lagrangian method to yield a sharp correlation peak.

On the other hand, colour provides much more information than intensity. The RGB colour space is the dominant colour space and the most frequently used. However, empirical evidence suggests that distances in colour spaces such as YIQ space correspond to perceptual colour differences more closely than do distances in RGB space. We will take advantage of this feature for colour pattern recognition. Our technique involves monochrome lasers for illumination and therefore, requires the color components $(Y, I$ and $Q)$ to be converted into gray level. 


\section{Analysis}

Color plays a role in object recognition. A color space is an arrangement of a coordinate system where each color is specified by a single point. RGB color space has been most frequently used. It consists of the red, green and blue respectively. However, RGB color model is not the most suitable color model on many applications. In this paper, the color seperation to design the reference function (or template) is based on YIQ color space, which stores color information in three channels, just like RGB. Y stands for luminance that represents the achromatic (black and white) image without any color. I and $Q$ convey colour information. $I$ is deviations from orange-luminance to cyan-luminance and $Q$ is deviations from purple-luminance to chartreuse-luminance. Moreover, the optoelectronic system is based on a MZJTC structure [8]. It is consisted of one laser, one spatial filter, one collimated lens (CL), 3 beam splitters (BSs), 3 polarizing beam splitters (PBSs), 3 Fourier lenses (FLs), 3 reflective liquid spatial light modulators (RLCSLMs), 3 charge coupled device (CCD) cameras, 1 electronic subtractor (ES) which is used for removing the zero-order term at the final output, and 1 computer for controlling the whole system. Besides, there are 1 half wave plate (HWP) and 1 quarter wave plate (QWP) in front of each of 3 RLCSLMs. The MZJTC structure is based on the Mach-Zehnder interferometer technique with Stokes relationships. The difference between conventional NOJTC and MZJTC is that the MZJTC structure needs only one step to remove the zero-order term. The processes are presented as follows.

First, 3 colour channels of the test colour image are jointly displayed in grayscale at one reflective liquid crystal spatial light modulator (RLCSLM). Similarly, 3 colour channel of the test colour image are also displayed in grayscale at another RLCSLM. The target on the first RLCSLM is illuminated and Fourier optically transformed. After passing through the beam splitter, the irradiance of transmitted and reflected Fourier spectrum is respectively detected by 2 CCDs in the Fourier frequency domain. Then, the difference of joint Fourier power spectrum between 2 CCDs is displayed at the third RLCSLM, such that the zero-order term will be subsequently removed at the output. Finally, the third CCD captures another Fouier transform spectrum of the difference. The output contains the overlapping of each cross-correlation of the reference channel and the target channel. More detailed analysis of MZJTC can be found in the literature [6-8].

For each of $\mathrm{Y}, \mathrm{I}, \mathrm{Q}$ channels, we minimize the average cross correlation energy by using the Lagrange multipliers method to suppress sidelobes and maintain the correlation peak at a specified height. This results in a closed form solution in the frequency domain. It is a column vector, which needs to be reordered back into a 2-D array, and then to be inverse Fourier transformed to the space domain.

To evaluate the recognition capability, some measurement criteria [13] including correlation peak intensity (CPI) and peak-to-correlation energy (PCE) are utilized. CPI is the cross-correlation peak intensity at the correlation output plane. PCE is defined as the energy of the peak correlation normalized to the total energy of the cross correlation plane.

\section{Result}

We use the image of one colourful insect as the basic pattern of the target. The size of the target is 64 $\times 64 \times 3$ pixels with intensity values in the range $[0,1] . Y, I$ and $Q$ channels are separated. For the purpose of comparison, another insect is selected as the nontarget. These two images are shown in Figure 1. For simplicity, We rotate these 2 objects in plane from $-14^{\circ}$ to $14^{\circ}$, and select patterns $2^{\circ}$ apart. Totally there are 15 rotationally distorted patterns per object used as the training set for each colour channel. Next, for each training set, we utilize the method of Lagrange multipliers for cross correlation optimization to obtain the reference template. 

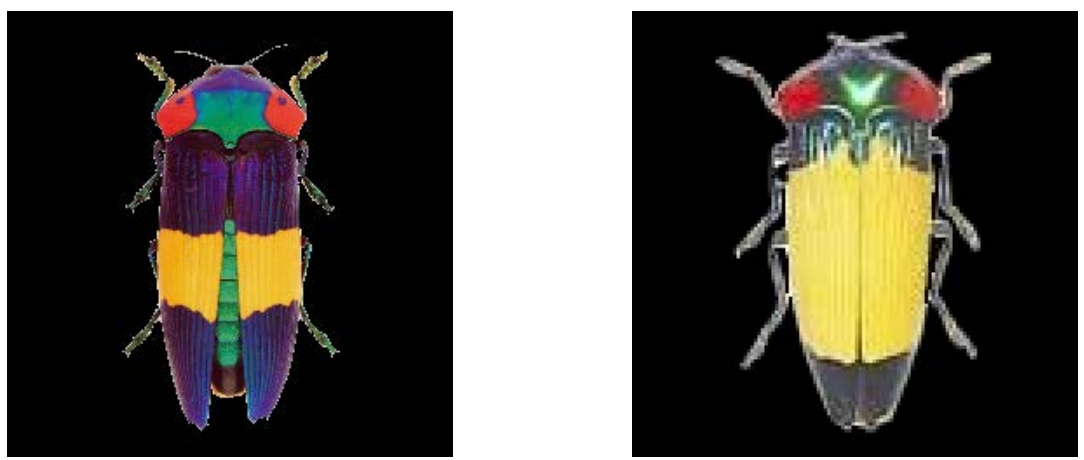

Figure 1: Target (left) and nontarget (right)
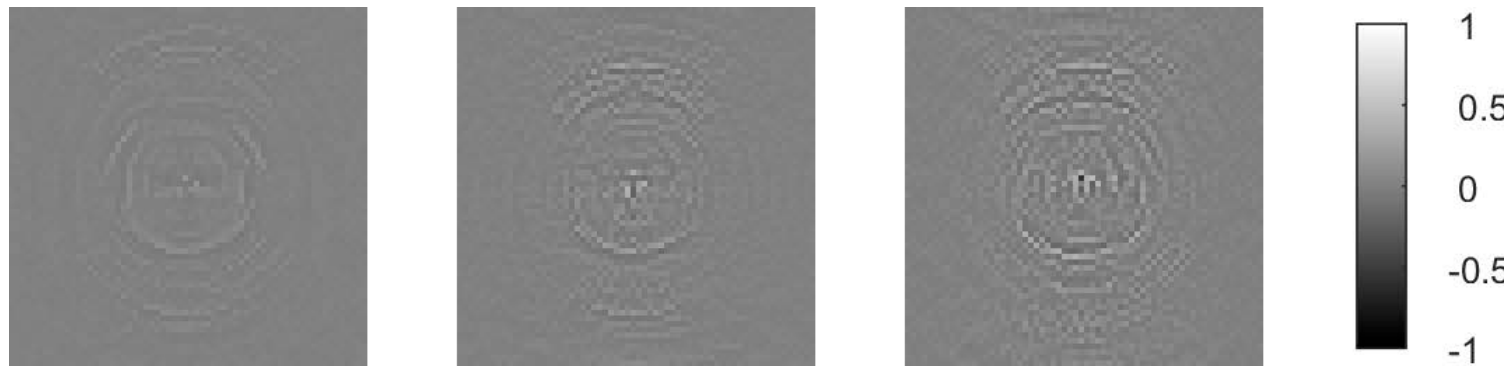

Figure 2: Y, I, Q reference functions for the target

Figure 2 shows respectively the synthesized reference function of $Y, I, Q$ channels from left to right. To cope with the optical requirement, the value of the grayscale is confined between -1 to 1 , as illustrated by the dynamic range on the right-hand side of the figure. It is worth noting that the CPI is designed to be the same for all training targets.

In Figure 3, the left image shows 3 color channels from one target displayed on one RLCSLM, while the right image shows 3 calculated reference functions displayed on another RLCSLM. The overall size of the joint image on each RLCSLM is $512 \times 512$ pixels. In Figure 4, the left image shows 3 color channels from one non-target displayed on one RLCSLM, while the right image shows calculated reference functions displayed on another RLCSLM.

The overall rotation invariance result is obtained, as illustrated in Figure 5. The CPI curve versus the rotation angle for the target as well as for the nontarget are shown in for the purpose of comparison. These 2 curves are seperated considerably. To determine whether the object under test is the target, we can set a threshold value of correlation peak, above which the input can be treated as a target and below which it is a non-target. Figure 6 illustrates three-dimensional plots of the output correlation intensity around the region of interest, where addition of desired cross correlations between the reference and the colour channel from all 3 channels occurs. Both the target and nontarget are $0^{\circ}$ rotated. As expected, the correlation peak is very narrow. Sidelobes suppression is achieved. High correlation peak corresponds to the correct pattern, whereas low correlation profile is observed for the nontarget. We obtain recognition of target and discrimination of nontarget. To see how much YIQ space improves, PCE curve for RGB colour space is also plotted in figure 7. The curve is lower than that for YIQ curve at each rotation angle of the target. The main reason is that, in most cases, when compared with RGB channels, YIQ channels are less correlated with other. This explains why the correlation profile is sharper for YIQ colour space. This reveals that by converting the RGB representation to the YIQ representation, the resulting three channels are less correlated and give better performance. 
Chulung Chen, Kaining Gu, J ianshuen Fang, Chungcheng Lee; Distortion Invariant Object Recognition Based on Mach-Zehnder J oint Transform Correlation in YIQ Colour Space. Advances in Image and Video Processing, Volume 7 No 1, February (2019); pp: 13-18
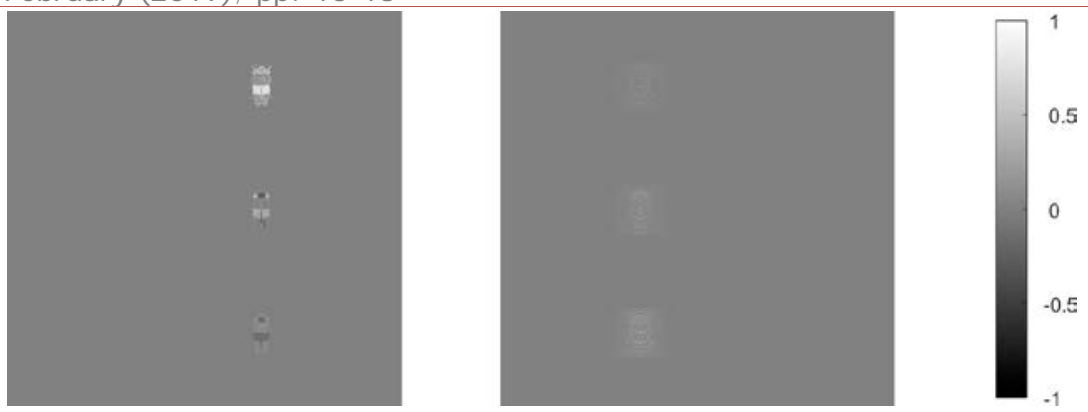

Figure 3: Left: 3 color channels from one target displayed on one RLCSLM; right: 3 calculated reference functions displayed on another RLCSLM.
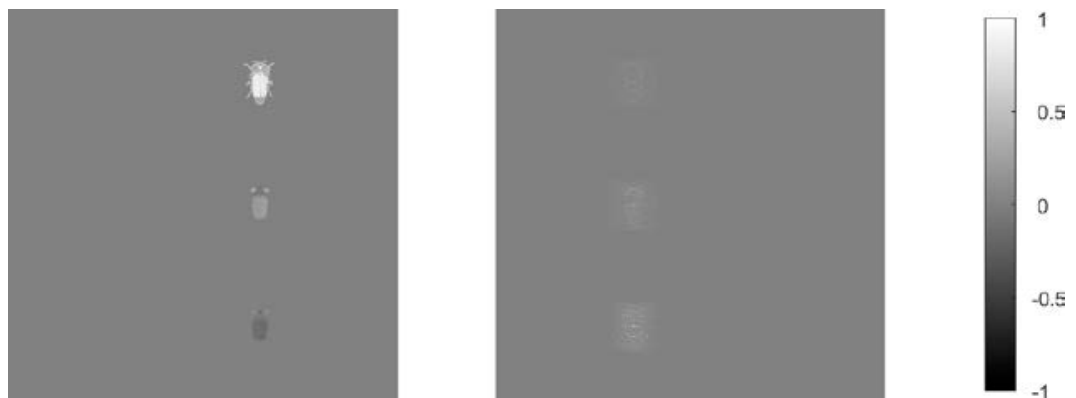

Figure 4: Left: 3 color channels from one non-target displayed on one RLCSLM; right: 3 calculated reference functions displayed on another RLCSLM.

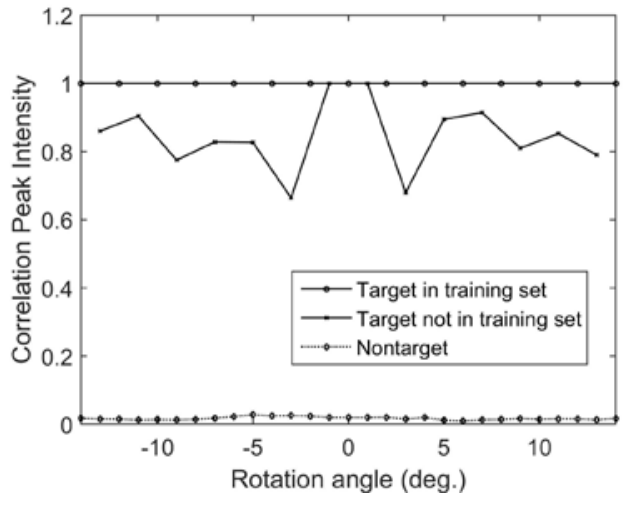

Figure 5: CPI versus rotation angle

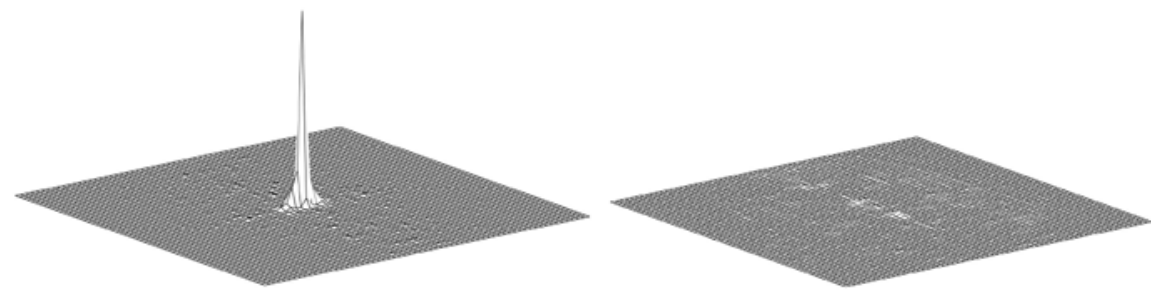

Figure 6: Example of correlation output for target (left) and nontarget (right). 


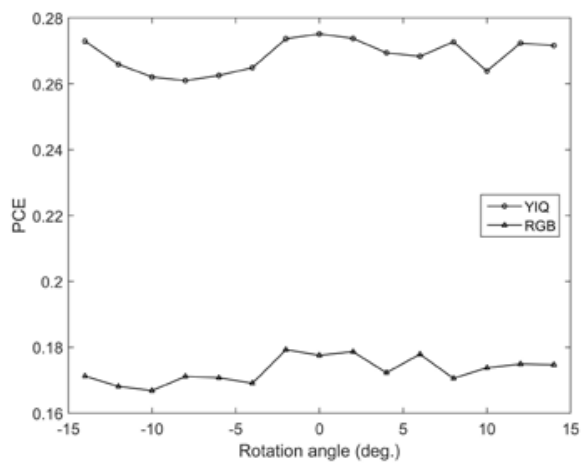

Figure 7: Comparison of the PCE between YIQ and RGB colour spaces as the target rotates.

\section{Conclusion}

In this research, we have provided an analysis of the performance of YIQ colour space together with average cross correlation optimization algorithm for pattern recognition on MZJTC. Comparison between YIQ and RGB colour spaces has been evaluated in terms of PCE. The improvement is remarkable. Numerical result shows that YIQ space outperforms conventional RGB space. It is exactly what we expected to see. This work is expected to provide valuable data for further research.

\section{REFERENCES}

[1] VanderLugt, A., Signal detection by complex spatial filtering. Information Theory, IEEE Transactions on, 1964. 10(2): p. 139-145.

[2] Weaver, C. S. and J. W. Goodman, A technique for optically convolving two functions. Applied Optics, 1966. 5: p. 1248-1249.

[3] Yu, F. T. S. and X. J. Lu, A real-time programmable joint-transform correlator. Optics Communications, 1984. 52: p. 10-16.

[4] Lu, G., et al., Imentation of a non-zero-order joint-transform correlator by use of phase-shifting techniques. Applied Optics, 1997. 36: p. 470-483.

[5] Li, C., S. Yin and F. T. S. Yu, Nonzero-order joint transform correlator. Optical Engineering, 1998. 37: p. 58-65.

[6] Cheng, C. and $\mathrm{H}$. Tu, Implementation of a nonzero-order joint transform correlator using interferometric technique. Optical Review, 2002. 9: p. 193-196.

[7] Chen, C., et al., Application of simulated annealing for color pattern recognition to hybrid optoelectronic joint transform correlator. Advances in Image and Video Processing, 2015. 3(6): p. 1317.

[8] Chen, C., et al., Pattern Recognition Based on YIQ Colour Space with Simulated Annealing Algorithm and Optoelectronic Joint Transform Correlation. Advances in Image and Video Processing, 2016. 4(5): p. 17-22.

[9] Wu, C., C. Chen, and J. Fang, Linearly constrained color pattern recognition with a non-zero order joint transform correlator. Optics Communications, 2002. 214: p. 65-75. 
Chulung Chen, Kaining Gu, Jianshuen Fang, Chungcheng Lee; Distortion Invariant Object Recognition Based on Mach-Zehnder J oint Transform Correlation in YIQ Colour Space. Advances in Image and Video Processing, Volume 7 No 1, February (2019); pp: 13-18

[10] Chen C., and J. Fang, Optimal synthesis of a real-valued template for synthetic aperture radar pattern recognition. Microwave and Optical Technology Letters, 2002. 32(2): p. 91-95.

[11] Fu, S., et al., Application of simulated annealing for color pattern recognition to the optoelectronic correlator with liquid crystal device. The 2012 IAENG International Conference on Imaging Engineering. p.683-688.

[12] Liu, C., et al., Pattern recognition by Mach-Zehnder joint transform correlator with binary power spectrum," Proceedings SPIE 8559.

[13] Kumar, B. V. K. V. and L. Hassebrook, Performance measures for correlation filters. Applied Optics, 1990. 29: p. 2997-3006. 